\title{
PANORAMA GENERAL DE LAS CLASES REMOTAS MEDIADAS POR TECNOLOGÍA
}

\author{
Alberto Montalvo Castro* \\ Yeimmy Katherine Serrano León ${ }^{* *}$ \\ Universitaria Agustiniana \\ https://doi.org/10.14718/9789585133846.2021.5
}

\section{A manera de partida}

Para el 16 de marzo de 2020, con la llegada del coronavirus (COVID-19) al país, el Gobierno nacional promulgó normas que tuvieron impacto en instituciones de toda índole -académicas, comerciales, sociales y políticas-, con el fin de promover el denominado distanciamiento social, una acción destinada a controlar la propagación del virus.

Con este propósito, el Ministerio de Salud y Protección Social, mediante la Resolución 385 del 12 de marzo de 2020, declaró la emergencia sanitaria por causa del coronavirus COVID-19 hasta el 30 de mayo de 2020, y, mediante la Resolución 464 del 18 de marzo de 2020, la medida sanitaria obligatoria de aislamiento preventivo; medidas reafirmadas y ampliadas por posteriores resoluciones debido a la diseminación del virus en los pobladores.

Licenciado en Matemáticas y Física, docente del Departamento de Ciencias Básicas de la Universitaria Agustiniana.

https://orcid.org/my-orcid?orcid=0000-0002-6034-6981

* Cursante del Máster Universitario en Análisis y Visualización de Datos Masivos / Visual Analytics and Big Data. Especialista en Estadística y Licenciada en Matemáticas.

yeimmy.serrano@uniagustiniana.edu.co 
La declaración de emergencia mundial generó la suspensión de las actividades escolares (Luthra, 2020) en instituciones educación nacional, públicas y privadas, de nivel básico, medio y superior, formales y no formales, razón por la cual estas instituciones se vieron obligadas a garantizar el proceso de formación, pero con el apoyo de procesos mediados por las tecnologías como estrategia para compensar las acciones desarrolladas en la presencialidad (Portillo et al., 2020).

Por estas circunstancias, la rectoría de la Universitaria Agustiniana promulgó distintos comunicados con los cuales dio orientaciones para el proceso de formación para los programas de la institución, así como para las diferentes instancias de la comunidad educativa.

Estas orientaciones redireccionaron las actividades de formación en las diversas asignaturas que antes adelantaban su trabajo en espacios presenciales, razón por la cual el equipo docente del Departamento de Ciencias Básicas asumió las acciones y alternativas propuestas por la Institución para proceder en el proceso de enseñanza, dirigiendo a la medicación por las Tecnologías de la Información y la Comunicación (TIC).

Por lo expuesto, en la reflexión que se presenta en este capítulo se tuvo como objetivo identificar algunas maneras de ver las clases mediadas por las tecnologías de la información, esto desde organizaciones del orden internacional que orientan condiciones políticas, personales, a nivel internacional y nacionales, para arribar a la perspectiva de los estudiantes tras el inesperado y obligado cambio de enseñanza, ahora mediada por las TIc.

\section{Perspectivas previas al aislamiento}

Durante los últimos años del siglo xx y primeros del xxi, distintos estudios habían identificado que las Tic aportaban favorablemente a las condiciones socioeconómicas de los países en vía de desarrollo; en línea con esto, en ese tiempo se promovió la adopción de políticas que apoyaran estos hallazgos. De esta forma, en diversas regiones se había promovido la inversión y el desarrollo de infraestructura (véase Asongu \& Le Roux, 2017; Frey, 2005; Mann, 2004; Sahay, 2001; Walsham et al., 2007) a partir de acciones impulsadas por directrices de organismos internacionales como el Centro de Investigaciones para el Desarrollo (IDRC), la Organización de Cooperación y Desarrollo Económicos (OCDE), o la CEPAL, que en su documento propuesto para la xv Cumbre del Mecanismo de Diálogo y Concertación de Tuxtla (CEPAL, 2015) planteó que implementar las TIc, con los avances que esto implica, impulsa la productividad de la sociedad, "lo que permite el desarrollo de dinámicas competitivas que permiten reducir la desigualdad, por facilitar el acceso a servicios públicos como la educación, la salud y otros relacionados con la gestión gubernamental" (p. 56).

Este movimiento se generó gracias a que las tecnologías permiten reducir las brechas entre los sujetos y las situaciones que le circundan, de manera que ayudan a vincular el aula de forma virtual con el espacio de cada uno de sus partícipes, sin necesidad de producir un 
desplazamiento físico. Como lo plantea Inés Dussel (2020), se pude estar de forma simultánea en casa, en el aula, en la habitación.

A pesar de estas y otras múltiples recomendaciones que se han generado al respecto, no podemos desconocer que en países subdesarrollados se agudiza la desigualdad en el manejo de las тIC. Aunque se ha incrementado el uso de dispositivos electrónicos, el manejo de contenidos digitales y el uso de plataformas colaborativas, también se identifica un número significativo de ciudadanos con acceso limitado a las tecnologías, la innovación y, con ello, al conocimiento, lo cual termina promoviendo marginación y exclusión social —condición contraria a lo ocurrido en economías desarrolladas- (Hilbert, 2016; Notten et al., 2009).

Respecto a esto, Dussel y Quevedo (2010) indican que en las últimas dos décadas se han promovido planes y programas para la inclusión digital como parte de agendas políticas en distintas zonas de América Latina, como es el caso del Ceibal en Uruguay, Enlaces en Chile, el Proyecto Huascarán en Perú, el Programa Computadoras para Educar en Colombia, el Programa Integral Conéctate en El Salvador, las Escuelas del Futuro en Guatemala o el Plan de Inclusión Digital Educativa y Conectar Igualdad en Argentina. Con estas propuestas, como afirman Dussel y Quevedo (2010), se ha buscado vincular a las regiones en los planes de transformación educativa y cultural por medio del manejo y aprendizaje de las Nuevas Tecnologías de la Información.

En el contexto colombiano, encontramos políticas gubernamentales como el Plan Vive Digital 2010-2014, 2014-2018 y 2018-2022, con los cuales se ha buscado masificar el uso del internet, y con ello las Tic, para promover la inclusión digital y, según el Ministerio de Tecnologías de la Información y las Comunicaciones [MINTIC] (2014), "reducir la pobreza, generar empleo, y desarrollar soluciones para los problemas de los colombianos" (p.1).

El hecho de considerar la masificación de las tecnologías, y efectuarla, implica una acción para promover la transformación del sistema educativo, lo cual implica pensar un modelo educativo centrado en el estudiante, donde este sea protagonista, un actor partícipe y activo de la construcción del conocimiento por medio de actividades en escenarios propios para este desarrollo. De esta manera, el estudiante se tiene que involucrar en procesos de aprendizaje que pueden estar relacionados con proyectos colaborativos, que le conducen al intercambio de experiencias de formación propias de su área laboral, y que además le permitirán llegar a analizar situaciones particulares para plantear alternativas novedosas de solución (Messina \& Travieso, 2017).

Desde esta perspectiva, las TIC son consideradas un instrumento que permite mediar el proceso de formación desde una propuesta pedagógica que aporta al trabajo colaborativo y que fortalece acciones comunicativas orientadas a la construcción de saberes por medio de trabajos en equipo (Litwin, 2005). Para esto, según Carrasco (2009), "se requiere que el 
docente sea pieza clave en el uso adecuado de los medios tecnológicos, debe readaptar su metodología y tener capacidad de integrar tales recursos para obtener mejor provecho de su trabajo" (p. 211).

De esta manera, vemos cómo se potencia la denominada e-learning —o formación virtual—, la cual permite promover saberes a un importante número de estudiantes por medio de los denominados Cursos Online Masivos y Abiertos (мо0с), así como por procesos de formación centrados en actividades experimentales a distancia, como los denominados Laboratorios Virtuales (Virtual Labs) —que corresponden a materiales simulados informáticamente-, los laboratorios a distancia (Remote Labs) — con la manipulación de materiales a distancia—, y el tratamiento de datos experimentales (Dat) — que corresponde al tratamiento de datos-, que son resultados de una experiencia real, pero accesible en tiempo real (De Jong et al., 2013, 2014).

Para este momento, como agente de cambio, la universidad tiene la responsabilidad de fomentar una visión educativa apalancada por la formación integral del profesional. Como plantea Zabala (2007), la formación se debe concebir como algo "mucho más puntual y funcional, dirigido a la adquisición de habilidades específicas y vinculadas, normalmente, al mundo del trabajo" (p. 40). De este modo, el trabajo del mundo actual está relacionado profundamente con las habilidades tecnológicas y, por ende, con el uso de medios tecnológicos con potencia y versatilidad en el volumen de datos, todo lo cual le permite alcanzar un apropiado análisis, interpretación y comprensión de los fenómenos.

No obstante, incluir las TIC en el proceso de formación no garantiza aportar en la calidad e innovación de la misma (Cabero, 2007); se requiere transformar la instrucción y, con esto, el proceso de formación del docente. Como afirma Alba (2004), "la solo inclusión de las Tic conlleva a la falsa perspectiva de innovación" (p. 21).

Ahora bien, la inclusión de las Tic en la universidad, o en la institución educativa, implica la constitución de modelos pedagógicos novedosos acordes a los entornos medidos; deben contar con procesos de colaboración en el desarrollo de la formación, y a su vez con la disposición de contenidos digitales diversos (Castaño \& Palazio, 2007).

Teniendo esto en cuenta, se identifica desde hace años la necesidad de transformar el aula, donde el docente esté en la posibilidad y capacidad de organizarse y aprender a aprender de forma autónoma e independiente, lo que le permitirá transformar su conocimiento de forma permanente y generar modelos educativos novedosos apropiados a las condiciones o necesidades de la sociedad de la información y conocimiento. Como lo plantea la unEsco (2008), "para vivir, aprender y trabajar con éxito en una sociedad cada vez más compleja, rica en información y basada en el conocimiento, los estudiantes y los docentes deben utilizar la tecnología digital con eficacia" (p. 2). 
De acuerdo con Kemelmajer (2020), los principales actores del proceso educativo deben estar en disposición de transformar los modelos tradicionales y considerar roles participativos con el fin de hacer que las condiciones actuales de aislamiento generen cambios duraderos que impacten de forma apropiada y positiva al proceso de educativo, sin importar el nivel en el cual este se desempeñe.

Para finales de segunda década del siglo Xxi, las propuestas metodológicas apoyadas en plataformas son bastante diversas, como es el caso de las TED (Tecnología, Entretenimiento y Diseño) —acciones que empiezan a tomar relevancia en los espacios de formación-y los MOOc como espacios virtuales de formación utilizados por reconocidas instituciones como la Massachusetts Institute of Technology (мIт) o la Universidad de Harvard, que para el 2020 ya desarrollaban posgrados completos en línea ${ }^{5}$. Esto ya identifica la migración de los centros de formación a la nube, situación que implica la permanente adaptación de los diversos participantes del proceso de formación a los cambios, con el riesgo de quedar desplazados ante la innegable realidad.

\section{Durante el aislamiento}

El aislamiento social, preventivo y obligatorio no fue una estrategia exclusiva de Colombia. El mundo entero se vio obligado a ejecutar esta acción y, por ende, a incidir en el proceso de formación. Según la Organización de Estados Iberoamericanos para la Educación, la Ciencia y la Cultura (OEI, 2021),

Se calcula que en los 23 países de la comunidad iberoamericana miembros de la OEı hay 14 millones de niños y niñas en el nivel infantil que han visto interrumpidas sus clases, 46 millones en educación primaria, 44 millones en secundaria y más de 18 millones de estudiantes universitarios. En total, se han visto afectados más de 122 millones de estudiantes en Iberoamérica. (p. 1)

Esto nos lleva a considerar la diversidad de situaciones que se viven en cada país. Por ejemplo, en Argentina, a partir de la Resolución 104 de 2020, el Ministerio de Educación condujo a implementar la modalidad virtual para el desarrollo del calendario académico, lo que produjo variación en los contenidos mínimos de algunas áreas de formación. Este caso se dio en la cátedra de Tecnología Educativa del Profesorado y Licenciatura en Ciencias de la Educación de la Facultad de Humanidades y Ciencias de la Educación de la Universidad Nacional de La Plata, para lo cual se hizo uso del aula virtual del campus FAHCE, con el apoyo de videoconferencias (Travieso et al., 2020).

5 En plataformas como www.edx.org 
Por otra parte, con la universalización y mejora de la plataforma digital Crea, implementada en Uruguay, en el marco del Plan Ceipal en el año 2007, se facilitó la adopción de las condiciones académicas de la nueva realidad en el proceso de formación, lo que dio cuenta de una ventaja en la región por la adhesión de aproximadamente 254000 usuarios, tal como lo indica Leandro Folgar, presidente del Plan Ceibal (Urbana, 2020, marzo 26).

En Colombia, como se indicó previamente, la Universitaria Agustiniana no fue la excepción, pues como IEs comprometida con la formación integral de los estudiantes, desde hace años ha procurado el desarrollo de procesos de formación mediados por las tic, razón por la cual, al realizar los programas presenciales, adelantó también procesos de formación virtual desde la plataforma de Apoyo a la Presencialidad Uniagustiniana — que es parte de la política rectoral de 2015-, con la cual se buscó flexibilizar el proceso de enseñanza-aprendizaje de los programas académicos y educación continua de la Uniagustiniana, y con la cual se ha buscado apoyar a docentes y estudiantes en el manejo apropiado de plataformas tanto académicas como de investigación.

\section{Marco metodológico}

Fueron muchos los esfuerzos de las Instituciones de Educación Superior, y en su preocupación por evaluar las dinámicas académicas llevadas a cabo en este proceso, el grupo de universidades de la Red de Departamentos de Ciencias Básicas se planteó la necesidad de evaluar la percepción de los estudiantes acerca de las clases mediadas por tecnología.

En este sentido, la Universidad Católica de Colombia creó el instrumento sobre el cual se recoge la información que se analiza a continuación y que fue ajustado a las necesidades de la Universitaria Agustiniana. Específicamente, el instrumentó fue diseñado y sometido a evaluación por pares durante el periodo 2021-1, y contó con la aplicación de una prueba piloto a una muestra de 222 estudiantes de diferentes programas académicos de pregrado de la Universidad Católica de Colombia que cursaban materias del componente básico.

En particular, el instrumento final incluyó información socioeconómica de los estudiantes - sexo, edad, ingresos económicos, estado laboral actual y recursos tecnológicos con los que cuenta—, información académica — jornada, programa y rendimiento académico-, y la percepción, evaluada en 10 preguntas en escala tipo Likert, de aspectos metodológicos, evaluativos y de uso de herramientas tecnológicas en las clases mediadas por tecnología.

La consistencia interna de los 10 ítems que componen el instrumento dio como resultado un coeficiente alfa de Cronbach de .825 , lo que da cuenta de una confiabilidad adecuada en la escala de valoración entre 1 ("Nunca") y 4 ("Siempre"). La población objeto de estudio 
fueron los estudiantes de la Universitaria Agustiniana que cursaron asignaturas del componente básico en los periodos 2020-1 y 2020-2, en las dos jornadas académicas.

Ahora bien, el análisis que se desarrolla para este capítulo es de tipo cuantitativo descriptivo y exploratorio, pues busca identificar la percepción que tienen los estudiantes del desarrollo de las clases mediadas por tecnología en estos dos periodos, así como establecer relaciones entre las variables descritas y realizar un comparativo entre periodos. La fuente principal de información estuvo constituida por las respuestas a la encuesta, difundida por medio de Google Forms durante los dos periodos académicos mencionados. El procesamiento de la información se llevó a cabo por medio del software estadístico SPSS, versión 26.

\section{Resultados, análisis y discusión}

En relación con el estudio, para el periodo 2020-1 se contó con la participación de 1774 estudiantes de la Universitaria Agustiniana que tomaron materias del componente básico y dieron respuesta a la encuesta de percepción; para el periodo 2020-2, la participación fue de 1262 estudiantes (véase Tabla 1).

Tabla 1: Distribución de las variables socioeconómicas por periodo

\begin{tabular}{|c|c|c|c|}
\hline \multirow{2}{*}{ Variable } & \multirow{2}{*}{ Grupo } & \multicolumn{2}{|c|}{ Individuos } \\
\hline & & $\%$ Total 2020-1 & $\%$ Total 2020-2 \\
\hline \multirow{2}{*}{ Género } & Hombres & 52 & 46 \\
\hline & Mujeres & 48 & 54 \\
\hline \multirow{2}{*}{ Jornada } & Diurno & 63 & 62 \\
\hline & Nocturno & 37 & 37 \\
\hline \multirow{2}{*}{ Trabajo } & Sí & 66 & 59 \\
\hline & No & 34 & 41 \\
\hline \multirow{4}{*}{ Ingresos } & Hasta 1 sMLV & 75 & 75 \\
\hline & Hasta 2 sMLV & 18 & 18 \\
\hline & Hasta $3 \mathrm{sMLV}$ & 4 & 4 \\
\hline & Más de 3 sMLV & 3 & 3 \\
\hline \multirow{3}{*}{$\begin{array}{l}\text { Rendimiento } \\
\text { académico }\end{array}$} & Bajo & 2 & 2 \\
\hline & Mediano & 40 & 35 \\
\hline & Óptimo & 59 & 63 \\
\hline \multirow{3}{*}{$\begin{array}{l}\text { Probabilidad } \\
\text { de continuar }\end{array}$} & Alta & 29 & 58 \\
\hline & Baja & 23 & 7 \\
\hline & Media & 49 & 35 \\
\hline \multicolumn{2}{|c|}{ Total } & 1774 & 1262 \\
\hline
\end{tabular}

Nota. SMLv: Salario mínimo legal vigente. 
Como se puede observar, la población estudiada se distribuyó casi de manera equitativa entre hombres y mujeres, con una leve variación en la tendencia de participación por género, pues mientras que en el 2020-1 el porcentaje de hombres fue de 52 \%, en el 2020-2 la población masculina disminuyó a un $46 \%$; siendo en este último periodo un menor porcentaje que en las mujeres.

Asimismo, los estudiantes se distribuyeron con la misma tendencia por jornada en los dos periodos analizados, con mayor porcentaje en la jornada diurna — 63\% y $62 \%$ para los periodos 2020-1 y 2020-2, respectivamente- en comparación con la jornada nocturna.

En la Figura 1 se discrimina a los estudiantes según el programa académico en curso. La mayor participación en los dos periodos se dio en el programa de Negocios Internacionales, con $24 \%$ en el 2020-1 y 22 \% en el 2020-2, seguido por el de Administración de Empresas, con $13 \%$ en ambos periodos, e Ingeniería Mecatrónica, con 13 \% en el 2020-2 y 10 \% en el 2020-1. La gran mayoría de los estudiantes encuestados hacía parte de la Facultad de Ciencias Económicas y Administrativas — 66 \% en el primer periodo y 64 \% en el segundo-.

Figura 1: Distribución de la población estudiantil en los programas académicos por periodo

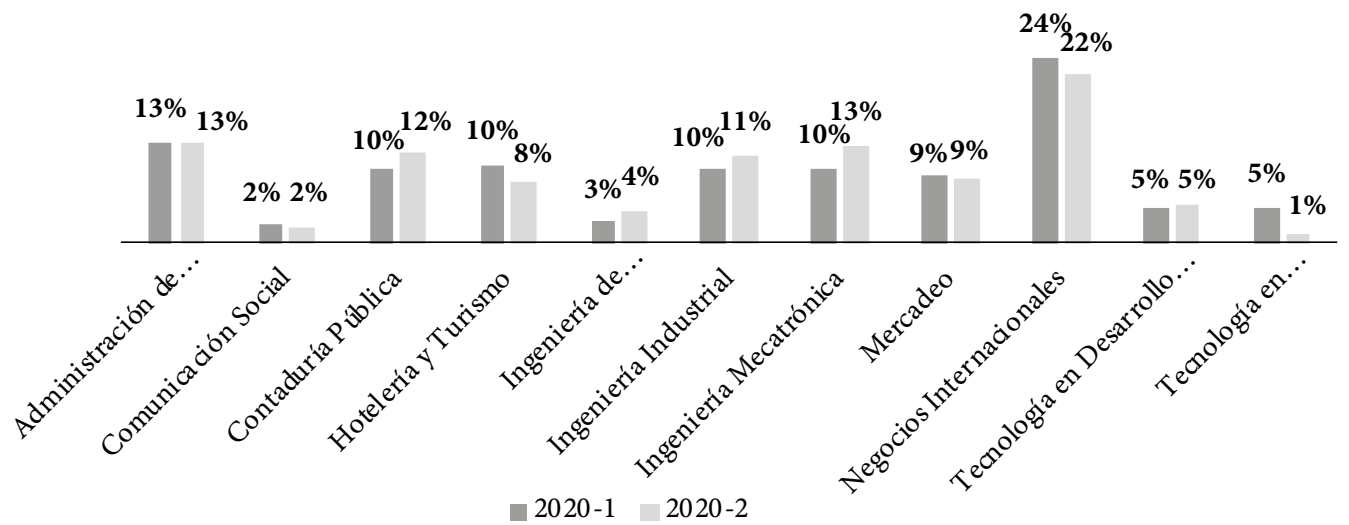

Con respecto a la situación económica, se observa que la población trabajadora disminuyó para el segundo periodo analizado. Como se puede observar, el porcentaje de estudiantes que trabajaba en el 2020-2 descendió de 66 \% a 59 \% en comparación con el 2020-1, mientras que los ingresos permanecieron constantes en ambos periodos: un $75 \%$ de estudiantes con ingresos hasta de un SMLV, $18 \%$ hasta dos SMLV, $4 \%$ hasta tres SMLV, y $3 \%$ más de tres SMLv. En el discriminado por género (véase Figura 2), se observa que a medida que los ingresos van aumentando, la brecha por género se va haciendo más notoria; es así que, hasta ingresos de un SMLv, la proporción de hombres y mujeres es semejante — con una diferencia del 4 \%-, mientras que con ingresos mayores a tres smLv la brecha aumenta a $22 \%$. 
Figura 2: Distribución de ingresos por género

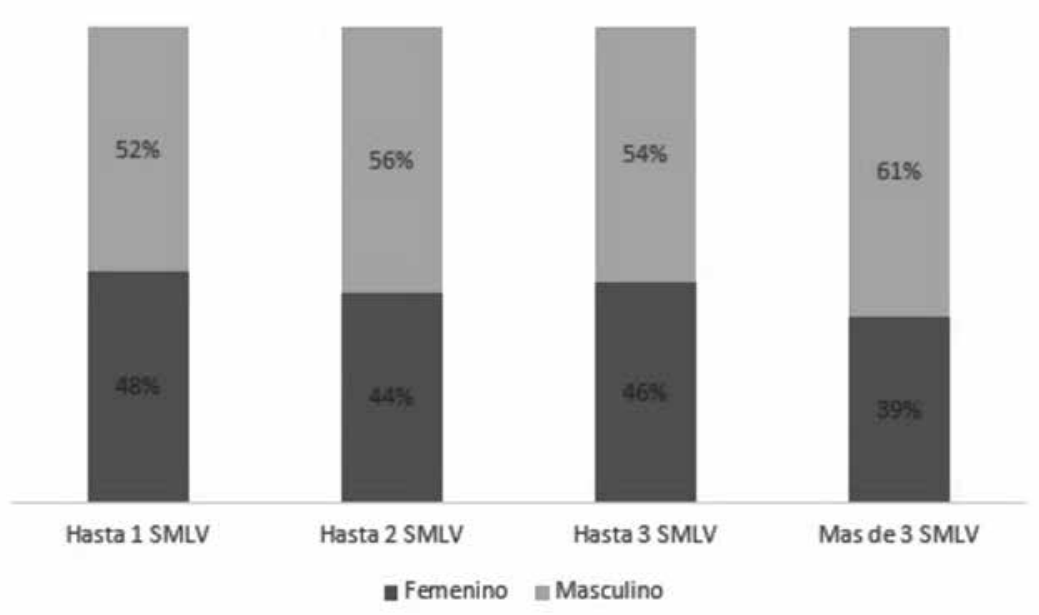

En la pregunta relacionada con la percepción que tiene el estudiante de su rendimiento académico, la gran mayoría consideró que es su rendimiento era "óptimo", con una apreciación de 59 \% en el 2020-1 y 63 \% en el 2020-2. De acuerdo con el género (véase Figura 3), mientras que es igual la percepción "óptima" del rendimiento en hombres y mujeres (49 \% y $51 \%$, respectivamente), más hombres consideraron un rendimiento "mediano" y "bajo" (58\% y $78 \%$, respectivamente) que mujeres (42\% y $22 \%$, respectivamente).

Figura 3: Distribución de desempeño por género

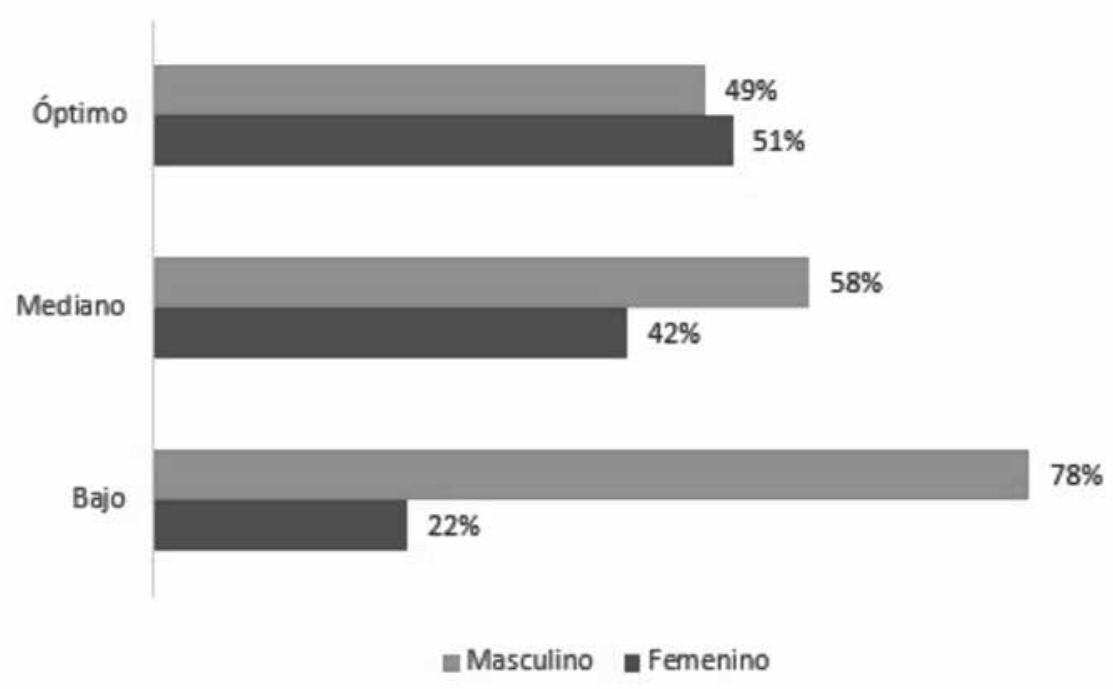


Ahora bien, analizando los resultados estadísticos de los ítems que evalúan la percepción de los estudiantes frente al desarrollo de las clases remotas para los periodos 2020-1 y 2020-2 (véase Tabla 2), se observa un comportamiento semejante en los dos periodos evaluados. La menor puntuación promedio fue para el ítem 2 ("La metodología ha permitido la construcción de conocimientos"), con 3.28 puntos en el 2020-1, mientras que para el periodo 2020-2 fue el ítem 6 ("Se ha hecho uso de diferentes recursos tecnológicos que facilitan el aprendizaje"), con 3.35 puntos, seguido por el ítem 3 ("Los contenidos programáticos se han presentado de manera adecuada, facilitando su aprendizaje"), con 3.32 puntos.

Tabla 2: Análisis descriptivo por ítem evaluado 2020-1 y 2020-2

\begin{tabular}{|l|c|c|c|c|}
\hline \multicolumn{1}{|c|}{ Estadísticos descriptivos } & \multicolumn{2}{|c|}{$2020-1$} & \multicolumn{2}{c|}{$2020-2$} \\
\cline { 2 - 5 } & Media & DE & Media & DE \\
\hline 1. Se ha cumplido con los horarios de las sesiones de clases. & 3.66 & 0.567 & 3.78 & 0.490 \\
\hline $\begin{array}{l}\text { 2. La metodología ha permitido la construcción de } \\
\text { conocimientos. }\end{array}$ & 3.28 & 0.726 & 3.45 & 0.692 \\
\hline $\begin{array}{l}\text { 3. Los contenidos programáticos se han presentado de manera } \\
\text { adecuada, facilitando su aprendizaje. }\end{array}$ & 3.32 & 0.715 & 3.46 & 0.683 \\
\hline $\begin{array}{l}\text { 4. Se han generado espacios de participación durante el desarro- } \\
\text { llo de las sesiones. }\end{array}$ & 3.54 & 0.667 & 3.68 & 0.581 \\
\hline $\begin{array}{l}\text { 5. Se han resuelto las dudas en las sesiones de clase o en su } \\
\text { defecto en las tutorías y atención a estudiantes }\end{array}$ & 3.57 & 0.648 & 3.66 & 0.595 \\
\hline $\begin{array}{l}\text { 6. Se ha hecho uso de diferentes recursos tecnológicos que } \\
\text { facilitan el aprendizaje. }\end{array}$ & 3.32 & 0.754 & 3.35 & 0.761 \\
\hline $\begin{array}{l}\text { 7. Los valores propios de la educación y de la Universidad } \\
\text { Católica se ven reflejados en las sesiones de clase. }\end{array}$ & 3.51 & 0.679 & 3.66 & 0.591 \\
\hline $\begin{array}{l}\text { 8. Se hace uso de los recursos del Aulas Virtuales de Apoyo a la } \\
\text { Presencialidad (Ava) en las sesiones de clase. }\end{array}$ & 3.56 & 0.677 & 3.63 & 0.627 \\
\hline $\begin{array}{l}\text { 9. La comunicación ha sido eficiente entre el docente y el } \\
\text { estudiante a través de las diferentes plataformas, Ava, correo } \\
\text { electrónico u otros medios tecnológicos. }\end{array}$ & 3.50 & 0.682 & 3.58 & 0.653 \\
\hline $\begin{array}{l}\text { 10. Las evaluaciones realizadas son coherentes con el desarrollo } \\
\text { de los contenidos programáticos en las sesiones remotas. }\end{array}$ & 3.64 & 0.595 & 3.69 & 0.559 \\
\hline N válido (por lista) & \multicolumn{2}{|c|}{1774} & \multicolumn{2}{|c|}{1262} \\
\hline
\end{tabular}

Por otro lado, los ítems que más valor medio presentaron fueron el ítem 10 ("Las evaluaciones realizadas son coherentes con el desarrollo de los contenidos programáticos en las sesiones remotas"), con 3.64 puntos en el primer periodo, y el ítem 1 ("Se ha cumplido con el acompañamiento docente"), con 3.78 puntos en el segundo periodo.

Finalmente, la puntuación media para cada uno de los ítems en los dos periodos se encuentra entre 3 y 4, lo que representa, en la especificación de la escala - 1 = "Nunca", 2 = "Algunas veces", 3 = "Casi siempre" y 4 = "Siempre"-, que los estudiantes evalúan los criterios que 
se cumplen entre "Casi siempre" y "Siempre", con una tendencia creciente en el segundo periodo, lo que indica que existió un proceso de aprendizaje y adecuación en la enseñanza por medio de la conexión remota.

\section{Análisis de correspondencias múltiples}

El análisis de correspondencias múltiples es una técnica de análisis multivariante de carácter exploratorio para variables categóricas. A partir de este análisis, valores con categorías similares se asocian, y los valores con categorías diferentes se alejan. Esta técnica normalmente es aplicada en estudios de las ciencias sociales, y entre las características más importantes se encuentra que permite descubrir interrelaciones entre las variables evaluadas, y que permite representar dicha información en gráficos bidimensionales para favorecer su comprensión (Quaglino \& Pagura, 1998).

En el mapa bidimensional se puede observar la distribución de las variables, y a partir de esto se reflejan las posibles relaciones existentes entre ellas. Allí, las categorías más cercanas al origen responden a las valoraciones con mayores frecuencias, mientras que las más alejadas son observaciones atípicas sobre el conjunto de datos.

Para el presente estudio, de acuerdo con la información que se representa y la forma de las asociaciones o relaciones entre variables para el periodo 2021-1 (véase Figura 4), se encuentra con mayor frecuencia que los estudiantes de ambos sexos, en condición laboral activa y no activa, y quienes califican su desempeño como "óptimo", percibieron cada uno de los ítems como "Siempre"; mientras que, con menor frecuencia, se puede identificar estudiantes que evalúan su desempeño como "medio", con una valoración a los ítems de "Casi siempre". Finalmente, entre los casos menos frecuentes se observan estudiantes que evalúan su desempeño como "bajo", y que han evaluado los ítems entre "Algunas veces" y "Nunca". 
Figura 4: Mapa de correspondencias para el periodo 2021-1

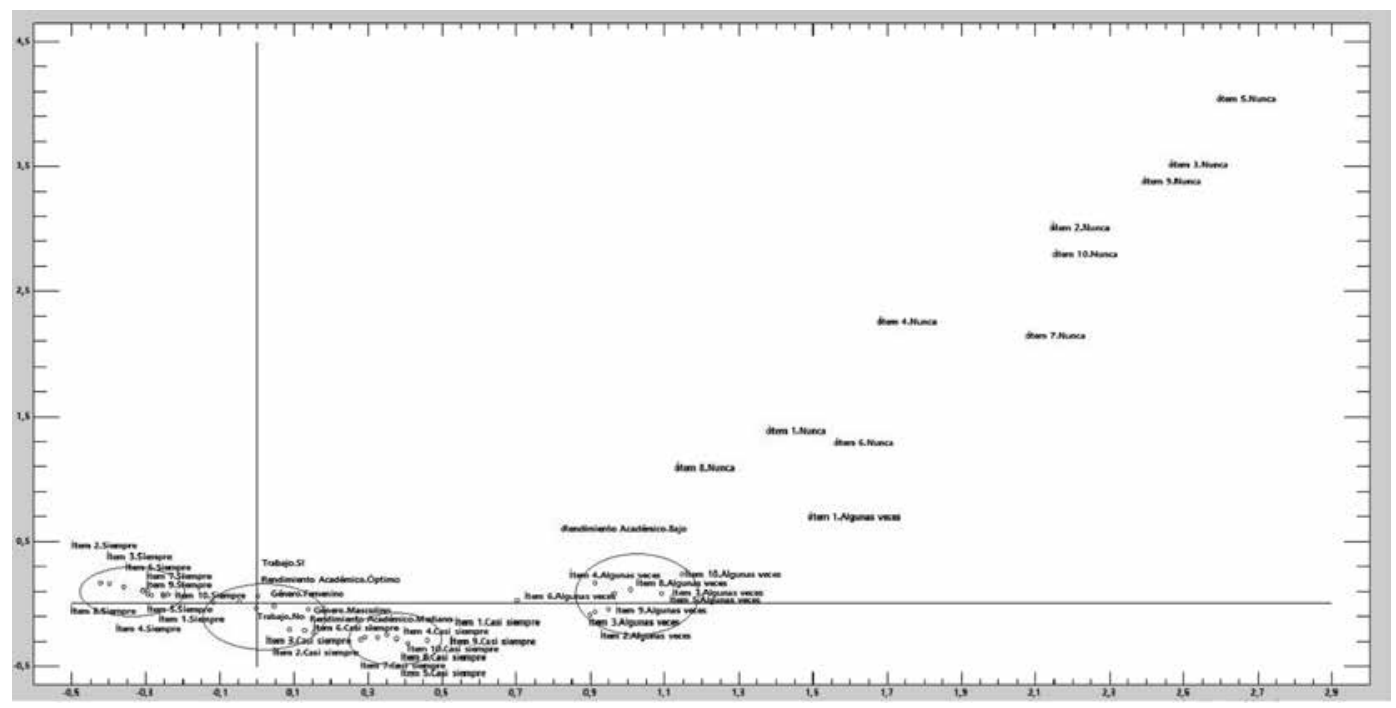

Por último, la Figura 5 muestra el comportamiento para el periodo 2021-2, muy semejante al periodo anterior, pues con mayor frecuencia se presentan hombres y mujeres en condiciones laborales activas y no activas que reconocen su desempeño como "óptimo" como los que han valorado los ítem que evalúan la percepción en diferentes criterios de las clases remotas como "Siempre". De igual manera, a medida que disminuye el rendimiento académico del estudiante, la percepción baja y se presenta con menor frecuencia. 
Figura 5: Mapa de correspondencias para el periodo 2021-2

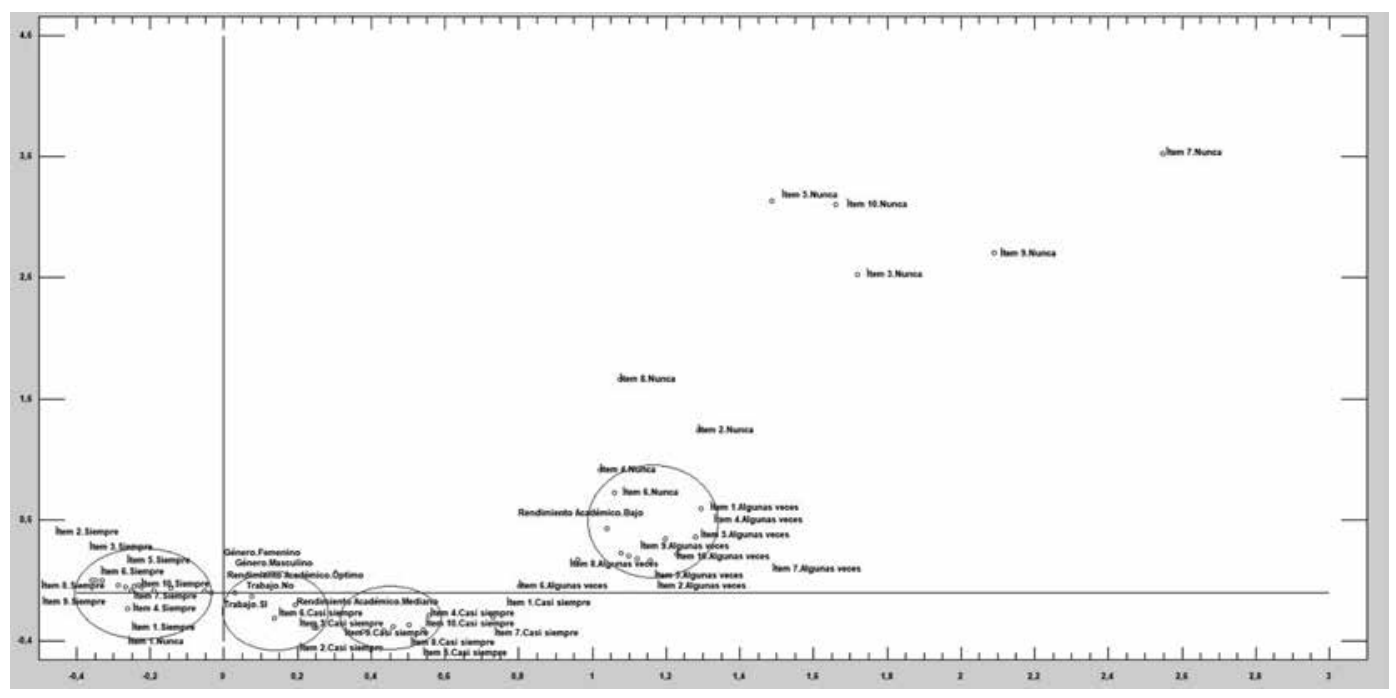

\section{Conclusiones}

Entre las características personales de los estudiantes se observa una distribución casi equitativa entre hombres y mujeres en los dos periodos. No obstante, debido a la crisis sanitaria que conllevó la crisis en otros aspectos del individuo a nivel social y económico, el aumento del desempleo se vio reflejado en la situación de los estudiantes, pues la población laboralmente activa disminuyó del 2020-1 al 2020-2. A nivel institucional, la mayoría de los estudiantes que cursaba el componente básico pertenecía a la jornada diurna en ambos periodos, con mayor participación de estudiantes de carreras de la Facultad de Ciencias Económicas y Administrativas.

Por otra parte, la valoración que daban los estudiantes respecto al desarrollo de las clases remotas mediadas por la tecnología determinó menores valores promedio para el desarrollo y aprendizaje de los contenidos programáticos, así como la contribución de la metodología desarrollada para la compresión de los temas y el uso de las herramientas tecnológicas, lo que proyecta que, a pesar de los esfuerzos de los docentes y directivos por ajustar la enseñanza presencial a una enseñanza mediada por tecnologías, aspectos relacionados con el uso de tecnologías, las metodologías de enseñanza y la apropiación de conceptos presentaron un menor rendimiento - pero sin ser deficientes, vale aclarar- No obstante, el esfuerzo de llevar una evaluación coherente a lo desarrollado y el acompañamiento docente fueron los aspectos mejor evaluados. 
De otro lado, se encontró una relación directa entre el rendimiento académico y la percepción que los estudiantes tuvieron respecto a las clases remotas, pues a medida que el desempeño de los estudiantes disminuye en la escala de "óptimo", "medio" y "bajo", la validación de los criterios también disminuyó en la escala de "Siempre", "Casi siempre", "Algunas veces" y "Nunca". A pesar de dicha tendencia, en términos generales se observa que con mayor frecuencia los estudiantes que consideran su desempeño como "óptimo" y "medio" también consideran que los criterios planteados en relación con las clases remotas se cumplen "Siempre" y "Casi siempre".

En síntesis, el panorama general de la situación muestra que para los dos periodos los estudiantes valoraron los esfuerzos por llevar a cabo las clases mediadas por tecnología, y esto ha favorecido su continuidad y proceso educativo. De hecho, para el 2021-2 la valoración se mostró semejante al periodo anterior, pero en tendencia creciente, lo que significa que el periodo 2021-1 puede ser considerado como un periodo de transición y adaptación que permitió consolidar los procesos.

\section{Referencias}

Alba, C. (2004). Viabilidad de las propuestas metodológicas para la aplicación del crédito europeo por parte del profesorado de las Universidades Españolas, vinculadas a la utilización de las TICs en la docencia y la investigación. Revista de educación, 337, 71-97. https://dialnet.unirioja.es/servlet/ articulo?codigo $=1271353$

Asongu, S. A., \& Le Roux, S. (2017). Enhancing ICT for inclusive human development in SubSaharan Africa. Technological Forecasting and Social Change, 118, 44-54. https://doi.org/10.1016/j. techfore.2017.01.026

Cabero, J. (2007). Las necesidades de las TIC en el ámbito educativo: oportunidades, riesgos y oportunidades. Tecnologías y educación educativas, 21(45). http://investigacion.ilce.edu.mx/tyce/45/ articulo1.pdf

Carrasco, J. B. (2009). Una didáctica para hoy. Cómo enseñar mejor. RIALP.

Castaño, C., \& Palazio, G. (2007). Nuevos escenarios pedagógicos a través de redes semánticas para el autoaprendizaje a lo largo de la vida (Life Long Learning). https://xdoc.mx/preview/ teoria-y-practica-del-aprendizaje-en-la-web-20-5de2cd7a36641

CEPAL. (2015). XV Cumbre del Mecanismo de Diálogo y Concertación de Tuxtla. CEPAL. https://repositorio.cepal.org/handle/11362/38426 
De Jong, T., Linn, M. C., \& Zacharia, Z. C. (2013). Physical and virtual laboratories in science and engineering education. Science, 340(6130), 305-308. https://doi.org/10.1126/science.1230579

De Jong, T., Sotiriou, S., \& Gillet, D. (2014). Innovations in STEM education: The GoLab federation of online labs. Smart Learning Environments, 1(3), 1-16. https://doi.org/10.1186/s40561-014-0003-6

Dussel, I. (2020). "La clase en pantuflas" Conversatorio virtual con Inés Dussel ISEP [Archivo de video]. Youtube. https://youtu.be/6xKvCtBC3Vs

Dussel, I., \& Quevedo, L. (2010). Educación y nuevas tecnologías: los desafíos pedagógicos ante el mundo digital. En VI Foro Latinoamericano de Educación. Santillana.

Frey, K. (2005). Gobernanza electrónica urbana e inclusión digital: experiencias en ciudades europeas y brasileñas. Nueva Sociedad, 196, 109-124. https://www.academia.edu/2977772/Gobernanza_electrónica_urbana_e_inclusión_digital_experiencias_en_ciudades_europeas_y_brasileñas

Hilbert, M. (2016). The bad news is that the digital access divide is here to stay: domestically installed bandwidths among 172 countries for 1986-2014. Telecommunications Policy, 40(6), 567-581. https:// doi.org/10.1016/j.telpol.2016.01.006

Kemelmajer, C. (2020). Educación en tiempos de pandemia: consejos de especialistas para enriquecer las aulas virtuales. Consejo Nacional de Investigaciones Científicas y Técnicas CONICET. https:// www.conicet.gov.ar/educacion-en-tiempos-de-pandemia-consejos-de-especialistas-para-enriquecerlas-aulas-virtuales/

Litwin, E. (Comp.). (2005). Tecnologías educativas en tiempos de internet. Amorrortu.

Luthra, P. (2020) Una ocasión para reinventar la escuela. Correo de la UNESCO: un solo mundo, voces múltiples. https://es.unesco.org/courier/2020-3/ocasionreinventar-escuela

Mann, C. L. (2004). Information technologies and international development: Conceptual clarity in the search for commonality and diversity. Information Technologies \& International Development, 1(2), 67-79. https://itidjournal.org/index.php/itid/article/download/146/146-482-1-PB.pdf

Messina, L., \& Travieso, M. (2017). El modelo educativo centrado en el estudiante. CEDU-IUPFA.

Ministerio de Tecnologías de la Información y las Comunicaciones. (2014). El Plan Vive Digital 20142018. MinTIC. https://mintic.gov.co/portal/vivedigital/612/w3-article-19654.html

Notten, N., Peter, J., Kraaykamp, G., \& Valkenburg, P. M. (2009). Digital divide across borders: A cross-national study of adolescents' use of digital technologies. European Sociological Review, 25(5), 551-560. https://doi.org/10.1093/esr/jcn071 
Organización de Estados Iberoamericanos para la Educación, la Ciencia y la Cultura (OEI). (2021, marzo 26). La OEI ofrece acceso libre a recursos educativos y culturales a administraciones públicas, docentes, estudiantes y familias. OEI. https://oei.int/oficinas/secretaria-general/noticias/la-oei-ofreceacceso-libre-a-recursos-educativos-y-culturales-a-administraciones-publicas-docentes-estudiantes-yfamilias

Portillo, S., Castellanos, L., Reynoso, O., \& Gavotto, O. (2020). Enseñanza remota de emergencia ante la pandemia Covid-19 en Educación Media Superior y Educación Superior. Propósitos y Representaciones, 8 (SPE3), e589. http://dx.doi.org/10.20511/pyr2020.v8nSPE3.589

Quaglino M., \& Pagura, M. (1998). Una propuesta para algunas aplicaciones de análisis de correspondencias múltiples. Terceras Jornadas de Investigaciones en la Facultad de Ciencias Económicas y Estadística (pp. 249-257). Universidad Nacional del Rosario, Argentina. https://core. ac.uk/download/pdf/90647111.pdf

Resolución 385 del 12 de marzo de 2020. Por la cual se declara la emergencia sanitaria por causa del coronavirus COVID-19 y se adoptan medidas para hacer frente al virus. Ministerio de Salud y Protección Social. $\quad$ https://www.minsalud.gov.co/sites/rid/Lists/BibliotecaDigital/RIDE/DE/DIJ/resolucion385-de-2020.pdf

Resolución 464 del 18 de marzo de 2020. Por la cual se adopta la medida sanitaria obligatoria de aislamiento preventivo, para proteger a los adultos mayores de 70 años. Ministerio de Salud y Protección Social. https://www.minsalud.gov.co/Normatividad_Nuevo/Resolucion\%20No.\%20464\%20del\%202020. pdf

Sahay, S. (2001). Introduction. Special issue on "IT and Health Care in Developing Countries". Electronic Journal on Information Systems in Developing Countries, 5(0), 1-6. https://doi. org/10.1002/j.1681-4835.2001.tb00029.x

Travieso, M., Zangara, A., Massini, M., López, M., \& Ivanoff, M. (2020). \#TecnoEduUNLP en tiempos de aislamiento: oportunidades y tensiones. InterCambios. Dilemas y transiciones de la Educación Superior, 7(2). http://dx.doi.org/10.2916/inter.7.2.12

UNESCO. (2008). Estándares de competencias en tic para docentes. https://eduteka.icesi.edu.co/ pdfdir/UNESCOEstandaresDocentes.pdf

Urbana. (2020, marzo 26). El plan ceibal en tiempos de coronavirus. Entrevista a Leandro Folgar. [Archivo de audio]. Urbana. https://urbana.uy/podcast/el-plan-ceibal-en-tiempos-de-coronavirus/

Walsham, G., Robey, D., \& Sahay, S. (2007). Special issue on information systems in developing countries. MIS Quarterly, 31(2), 317-326. https://www.uio.no/studier/emner/matnat/ifi/INF9200/v10/ readings/papers/WalshamRobeySahay.pdf

Zabala, A., \& Arnau, L. (2007). 11 ideas claves cómo aprender y enseñar competencias. Ed. Grao. 